\title{
A Study about Honey Bee Dance Serious Game for Kids Using Hand Gesture
}

\author{
Ok-Hue Cho and Sung-Tae Lee* \\ Seoul Cyber Univ., Mia-dong, Gangbuk-gu, Seoul, Korea 142-700, South Korea \\ pluszzang@hotmail.com, stlee@iscu.ac.kr
}

\begin{abstract}
Hand gesture is a form of non-verbal communication or non-vocal communication in which visible bodily actions communicate particular messages, either in place of, or in conjunction with, speech. Also, gesture is good interaction way when using domputer for kids or disabled. Especially, gesture is good educational way for kids. The can see many kinds of serious game using gesture. In this paper, we developed' serion game for kids using 'Leap Motion' based on honey bee dance. There are many gesture recognition ways as Kinect ${ }^{1}$, Leap Motion ${ }^{2}$ or Wii Remote ${ }^{3}$. These devices are easy interaction device also game controller. Our research is focused on hand gesture, so we used Leap Motion. Development of Leap Motion is affordable and convenient structure. After investigation of honey bee dance, we applied it into serious game. The dances are performed by worker bees that have returned to the honeycomb. It essentially is a languge that tells the other workers where the food is. In our developed game, kids can leanthese honey bee dance using their hands via Leap Motion. Leap Motion (is a comptter hardware sensor device that supports hand and finger motions as input, nalogous to mouse, but requiring no hand contact or touching. The movement of the hand is good for kid's brain development. It has already been proven through many materials. Kids canlearn the language of bees through developed game and it is a natural form of play. Our system can propose the new development direction of serious game, also potential of Lép Motion as serious game device.
\end{abstract}

Key Words: serious game, honey bee dance, Leap Motion

\section{Introduction}

A seriou game or applied game is a game designed for a primary purpose other than pure entertainment. The "serious" adjective is generally pretended to refer to products used by

\footnotetext{
* Corresponding Author

${ }^{1}$ Kinect (codenamed in development as Project Natal) is a line of motion sensing input devices by Microsoft for Xbox 360 and Xbox One video game consoles and Windows PCs. Based around a webcam-style add-on peripheral, it enables users to control and interact with their console/computer without the need for a game controller, through a natural user interface using gestures and spoken commands[1]

${ }^{2}$ Leap Motion, Inc. is an American company that manufactures and markets a computer hardware sensor device that supports hand and finger motions as input, analogous to a mouse, but requiring no hand contact or touching.

3 The Wii Remote (Japanese: Wii リモコン Hepburn: Wī Rimokon), also known colloquially as the Wiimote, is the primary controller for Nintendo's Wii console. A main feature of the Wii Remote is its motion sensing capability, which allows the user to interact with and manipulate items on screen via gesture recognition and pointing through the use of accelerometer and optical sensor technology.
} 
industries like defense, education, scientific exploration, health care, emergency management, city planning, engineering, religion, and politics. Educational game for kids is an active field in serious game. The term "serious game" has been used long before the introduction of computer and electronic devices into entertainment. Clark Abt discussed the idea and used the term in his 1970 book Serious Games, [2] published by Viking Press. In that book, his references were primarily to the use of board and card games. But he gave a useful general definition which is still considered applicable in the computer age.

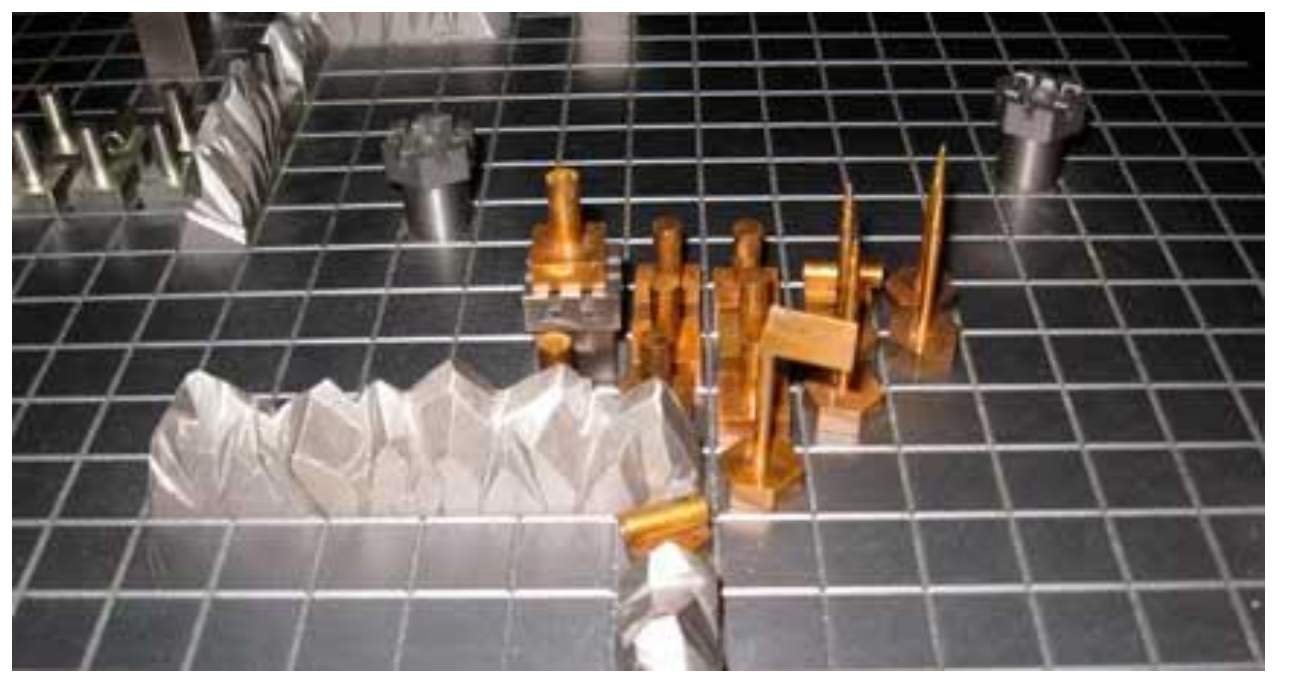

\section{Figure 1. Kriegsspiel (war game)}

Military officers have been using wavgames in order to train strategic skills for a long time. One early example of a serious game is a 19th-century Prussian military training game called Kriegsspiel as Fig 1, the German name for wargame. Mike Zyda provided an update and a logical approach to the term in his 2005 article in IEEE Computer entitled, "From Visual Simulation to Virtual Reality to Games". Zyda's definition begins with "game" and proceeds from there:

Serious Game: "a mental contest, played with a computer in accordance with specific rules that uses entertainment to further government or corporate training, education, health, public policy, and strategic communication objectives."

We developed serious game for kids. Normal kids cannot control computer well, so many educationat game contents are developed into easy and fun play form. In this, interaction device is mportant. Leap Motion is latest interaction device and low price, also setup and using are very easy especially to kids. We developed serious game for kids using 'Leap Motion' based on honey bee dance. Bee is Bees are flying insects closely related to wasps and ants, and are known for their role in pollination and for producing honey and beeswax. Bees are familiar with kids through fairy tales or toys and games. After investigation of honey bee dance, we applied it into serious game. The dances are performed by worker bees that have returned to the honeycomb. It essentially is a language that tells the other workers where the food is. In our developed game, kids can learn these honey bee dance using their hands via Leap Motion. Leap Motion is a computer hardware sensor device that supports hand and finger motions as input, analogous to a mouse, but requiring no hand contact or touching. Kids learn through play, so naturally they are immersed. In addition, the development of technology effects on game quite a lot and in a game interaction and 
immersion is also important elements. Janet H. Murray explained the 'Immersion' is a pleasant experience that going into exquisitely processed illusion fantasy world [3]. The immersion in game is important element to make the player continuously play the game [4]. On the 2nd chapter arrange the related works about Leap Motion and honey bee dance, in the 3 rd chapter we developed educational serious game and for the last 4th chapter arrange the conclusion and future study to finish the paper.

\section{Related Work}

\subsection{Leap Motion}

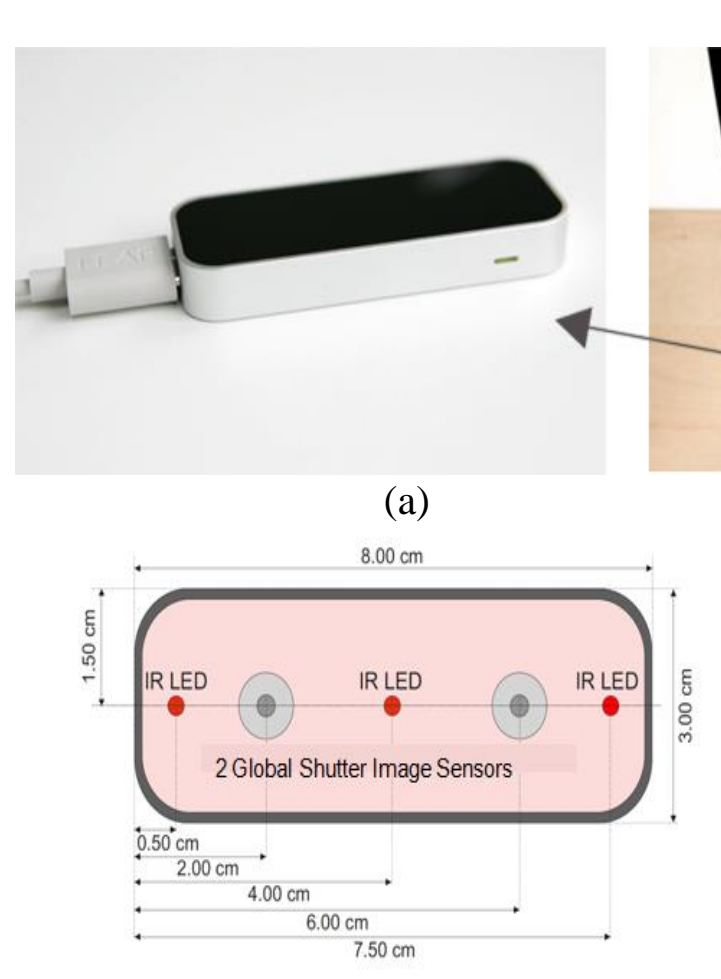

(c)

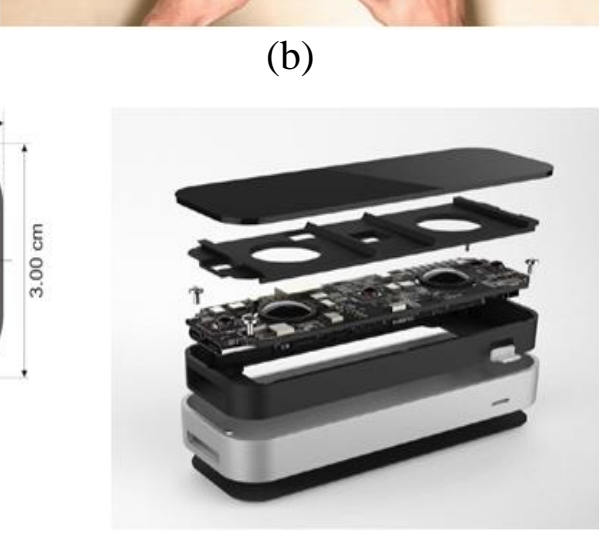

(d)

\section{Figure 1. Leáp Motion (a) Leap Motion pc Connect Test Image, (b) Structure of Leap Motion (c) (d)}

The,Leap Motion (Figure 1) controller is a small USB peripheral device which is designed to be placed on a physical desktop, facing upward. Using two monochromatic IR cameras and three infrared LEDs, the device observes a roughly hemispherical area, to a distance of about 1 meter (3 feet). The LEDs generate a 3D pattern of dots of IR light [5] and the cameras generate almost 300 frames per second of reflected data. The Leap Motion was shown to perform tasks such as navigating a website, using pinch-to-zoom gestures on maps, highprecision drawing, and manipulating complex 3D data visualizations [6]. 


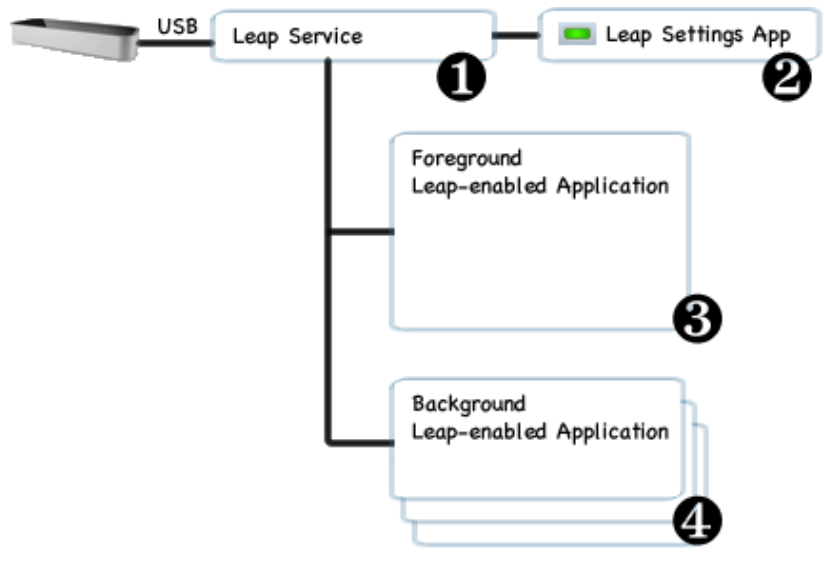

Figure 2. Leap-enabled Applications

The native application interface is provided throug $\alpha$ dynamically loaded library. This library connects to the Leap Motion service and provides tracking data to each application. We can link to the library directly in $\mathrm{C}++$ and Objective-C applications, or through one of the language bindings provided for Java, C\#, and Python [79. The Leap Motion service receives data from the Leap Motion Controller oyen the USB bus. It processes that information and sends it to running Leap-enabled applications. By default, the service only sends tracking data to the foreground application. However, applications can request that they receive data in the background as Figure 2 .

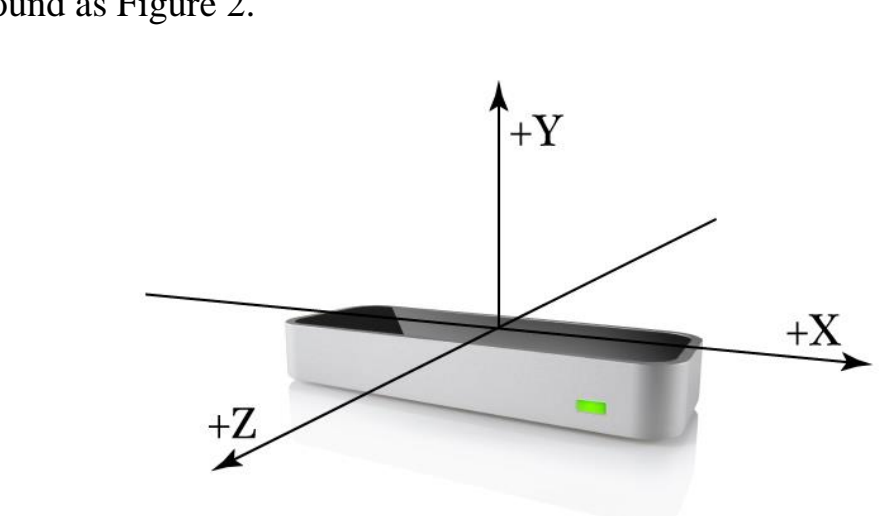

\section{Figure 3. The Leap Motion Right-handed Coordinate System}

The Leap Motion system has X, Y, Z dimensions. It employs a right-handed Cartesian coordinate system. The origin is centered at the top of the Leap Motion Controller. The Xand $\mathrm{z}$-axes lie in the horizontal plane, with the $\mathrm{x}$-axis running parallel to the long edge of the device and the y-axis is vertical, with positive values increasing upwards (in contrast to the downward orientation of most computer graphics coordinate systems). The z-axis has positive values increasing toward the user.

This device tracks hands, fingers, and tools in its field of view, it provides updates as a set, or frame, of data as Figure 4. Each Frame object representing a frame contains lists of the basic tracking data, such as hands, fingers, and tools, as well as recognized gestures and factors describing the overall motion in the scene. The Frame object is essentially the root of the Leap Motion data model. 

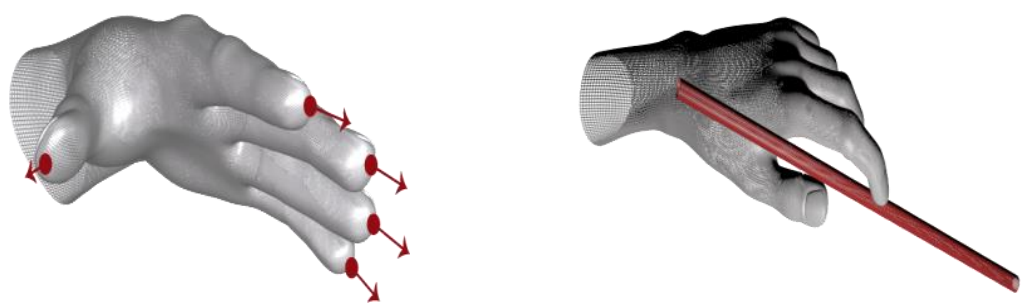

Figure 4. The Leap Motion Right-handed Coordinate System

Also it recognizes certain movement patterns as gestures which could indicate a use /ntent or command. Gestures are observed for each finger or tool individually. The Lleap Motion software reports gestures observed in a frame the in the same way that it reports other motion tracking data like fingers and hands as Figure 5.
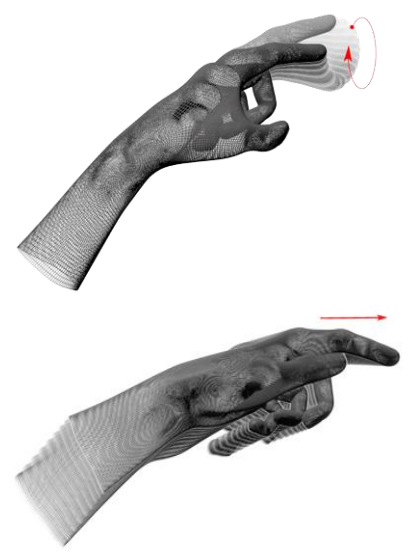

circle
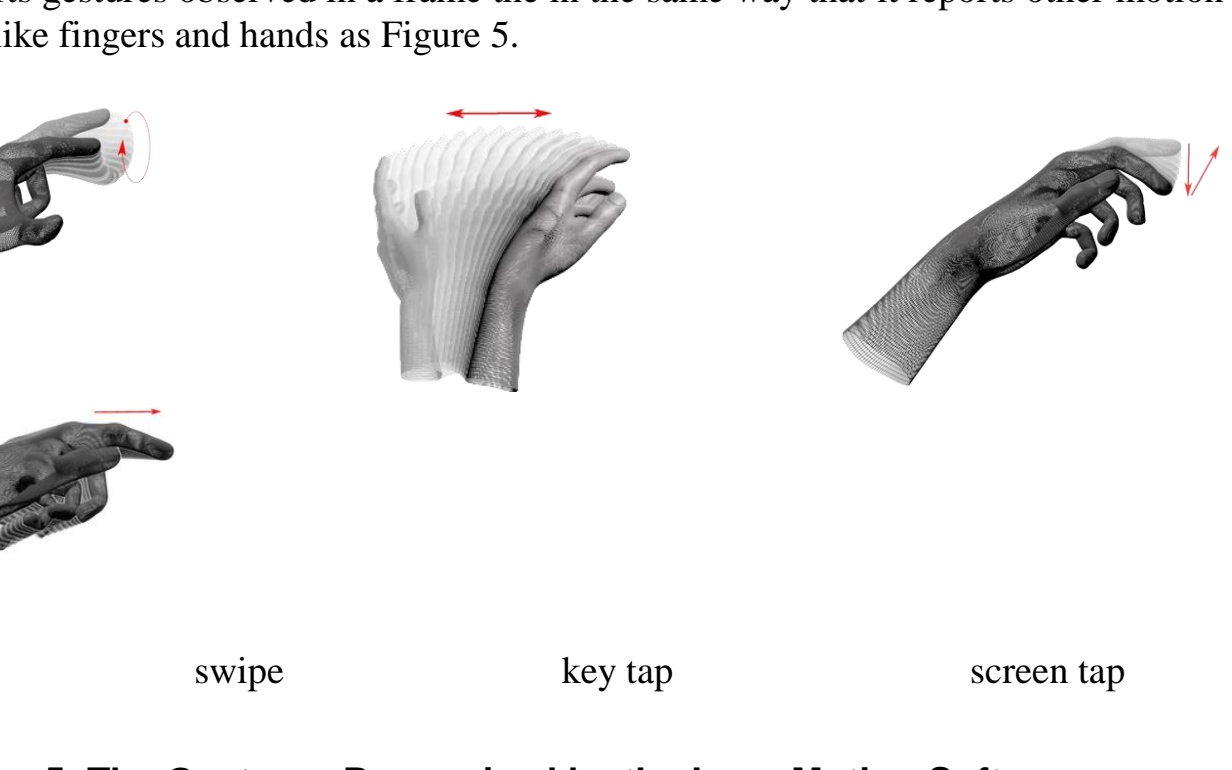

Figure 5. The Gestures Recognized by the Leap Motion Software

\subsection{Honey Bee Dance}

Our developed serious game is based on honey bee dance. Honey Bees appeared in a variety of multimedia content. It is very familiar insect to children. Biological characteristics of honey bees are very educational and scientific. Through our development serious game, kids can learn about honey bee dance language. There are two honey bee dances, round dace and waggle dance. Honey bee dace is the language of bee. Performed by a worker bee that has returned to the honey comb with pollen or nectar. It expresses telling to other workers where the food is. By signaling both distance and direction with particular movements, the worker bee uses the dance language to recruit and direct other workers in gathering pollen and nectar. When bee find a food source, and if it is very close to the hive (less than 50 meters), a forager performs a round dance as Figure 6, it is round dance. The waggle dance (Figure 6) is performed to express distance and direction of food to other worker bee, when food sources are more than 150 meters from the hive [8]. 

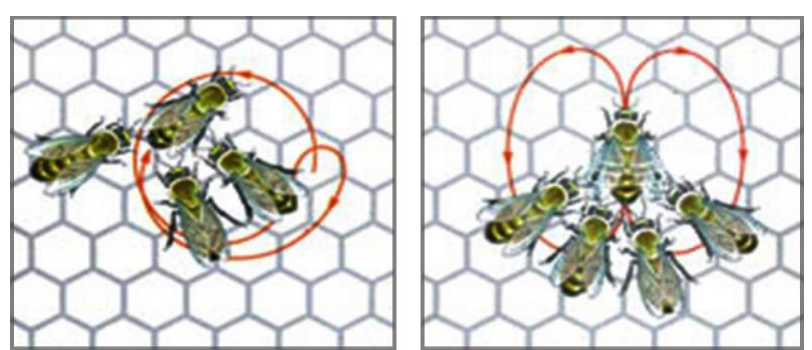

\section{Figure 6. Round Dance (left) and Waggle Dance (right) of Honey Bee}

\section{Development}

Gaming has been used in educational circles since at least the 1900s. Use of paper-based educational games became popular in the 1960s and 1970s, but waned under the Back to Basics teaching movement [9]. Through our development serious game, kids can learn about honey bee dance language. Developed specifications are as Table 1.

\section{Table 1. Game Development Specificakions}

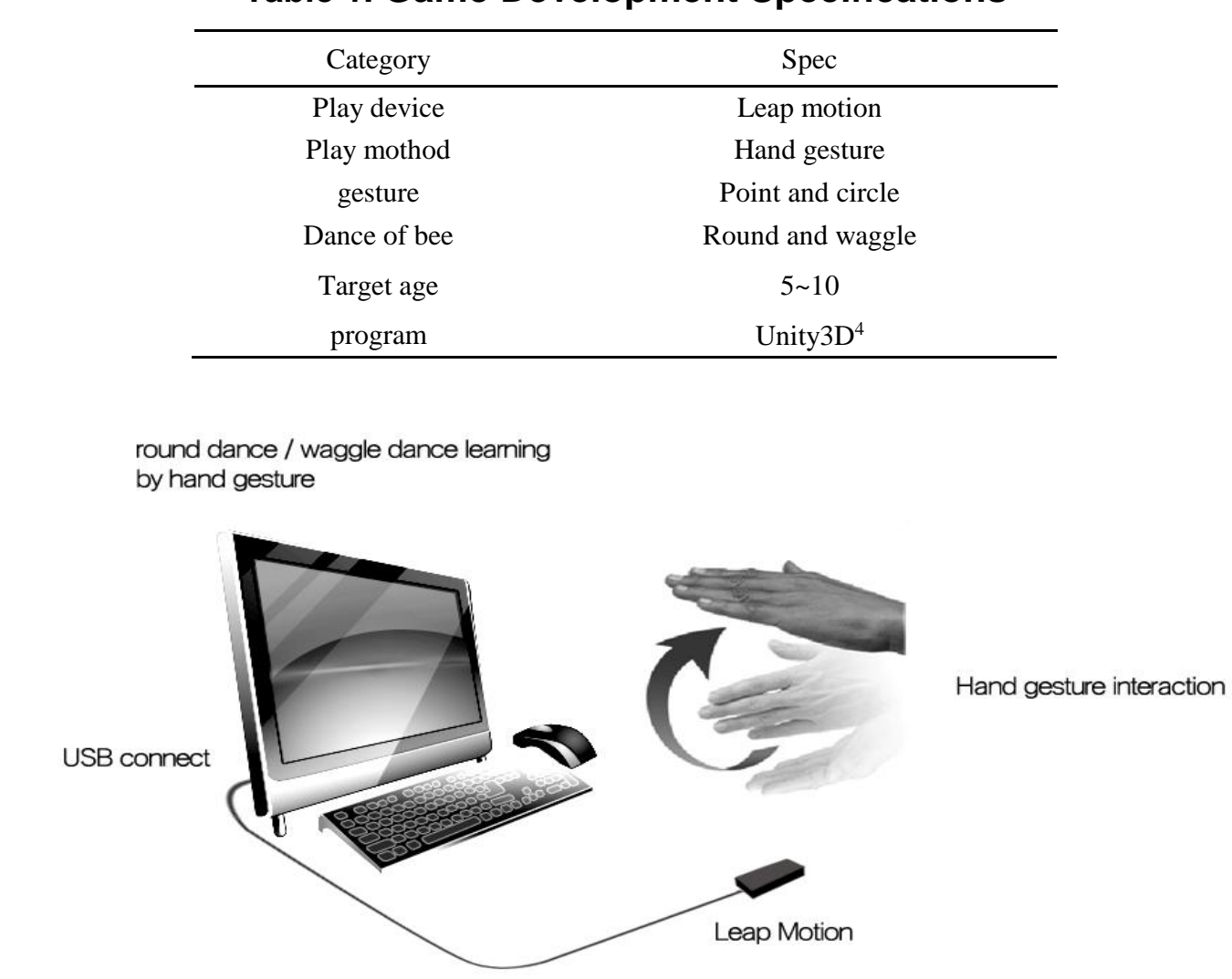

Figure 7. Game Play Flow Plan

\footnotetext{
${ }^{4}$ Unity is a cross-platform game engine with a built-in IDE developed by Unity Technologies. It is used to develop video games for web plugins, desktop platforms, consoles and mobile devices. It grew from an OS X supported game development tool in 2005 to a multi-platform game engine.[10]
} 
As Figure 7 player doesn't have to touch the computer. Play can play developed serious game only using hand gesture through the Leap Motion. Honey bee dances are language of bee. Kinds can learn and understand this language using their hand gesture.
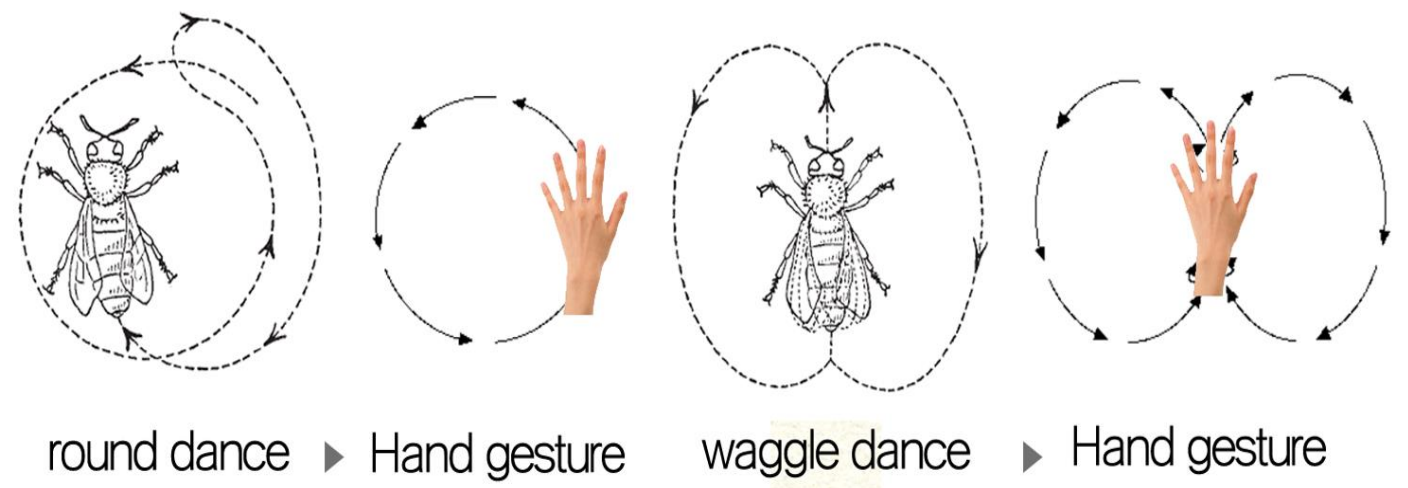

Figure 8. Each Dance and Hand Gesture in Developed Game

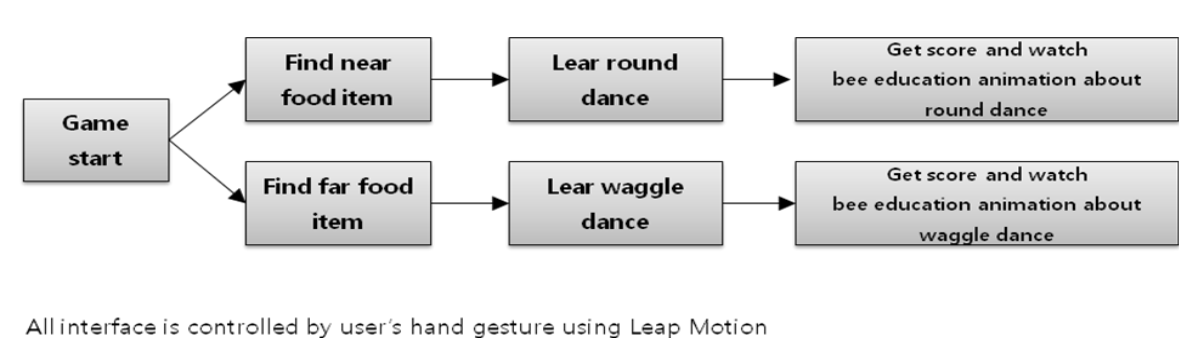

All interface is controlled by uger's hand gestare using Leap Motion
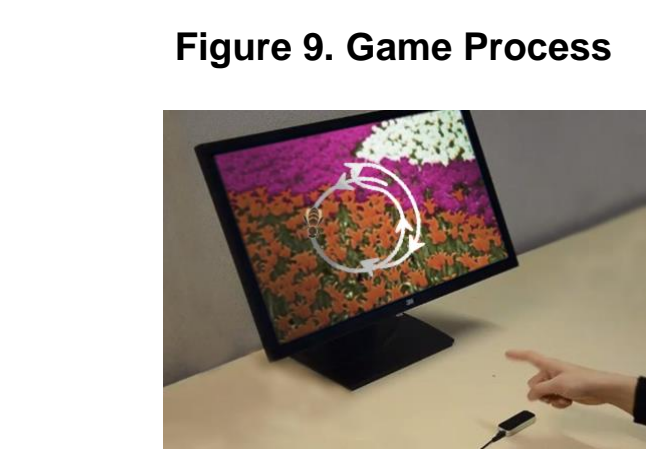

Figure 10. Test Image

Table 1 is game development specifications. We used Unity3D game engine to develop game. Point and circle gesture are recognized in our game. Target age is from 5age to 10age. Figure 7 shows game play flow plan and Figure 8 shows each dance and each hand gesture in developed game. Figure 9 shows game process. Figure 10 is test image.

\section{Conclusion}

We developed serious game for kids using 'Leap Motion' based on honey bee dance. After investigation of honey bee dance, we applied it into serious game. The dances are performed by worker bees that have returned to the honeycomb. The movement of the hand is good for kid's brain development. It has already been proven through many materials. Kids 
can learn the language of bees through developed game and it is a natural form of play. Our system can propose the new development direction of serious game, also potential of Leap Motion as serious game device. In future work, we will develop game to learn more detail ecological habit of bee for kids.

\section{References}

[1] "Project Natal" 101", Microsoft, (2009) June 1.

[2] Abt Associates Inc. History, 1970-1974, Abt Associates Inc., Retrieved: (2009) July 18.

[3] H. Yoonjeong and P. Seonho, "A Study on the Human and Interaction of actual space and virtural space by New media", Korean Research Foundation Scientific Learned Journal, vol. 06, no. 12, (2006), pp. 374.

[4] O.-H. Cho and W.-H. Lee, "Application of Reinforcement Learning System to Interactive Pigital Art", (Journal of internet technology), vol. 14, no. 1, (2013), pp. 99-106.

[5] http://www.youtube.com/watch?v=UI5EBzU_QqM.

[6] D. Terdiman, (2012-05-20). "Leap Motion: 3D hands-free motion control, unboand", Cutting Edge, c|net. Retrieved: (2012) May 23.

[7] https://developer.leapmotion.com/documentation/csharp/deyguide/Leap_Architecturd.html.

[8] D. R. Tarpy, "The Honey Bee Dance Language", North Carolina Cooperative Extension Service, (2004) April.

[9] J. W. Rice, "Assessing higher order thinking in video games. Journal of Technology and Teacher Education, vol. 15 , no. 1, (2007), pp. 87.

[10] Wikipedia, "Unity3D”.

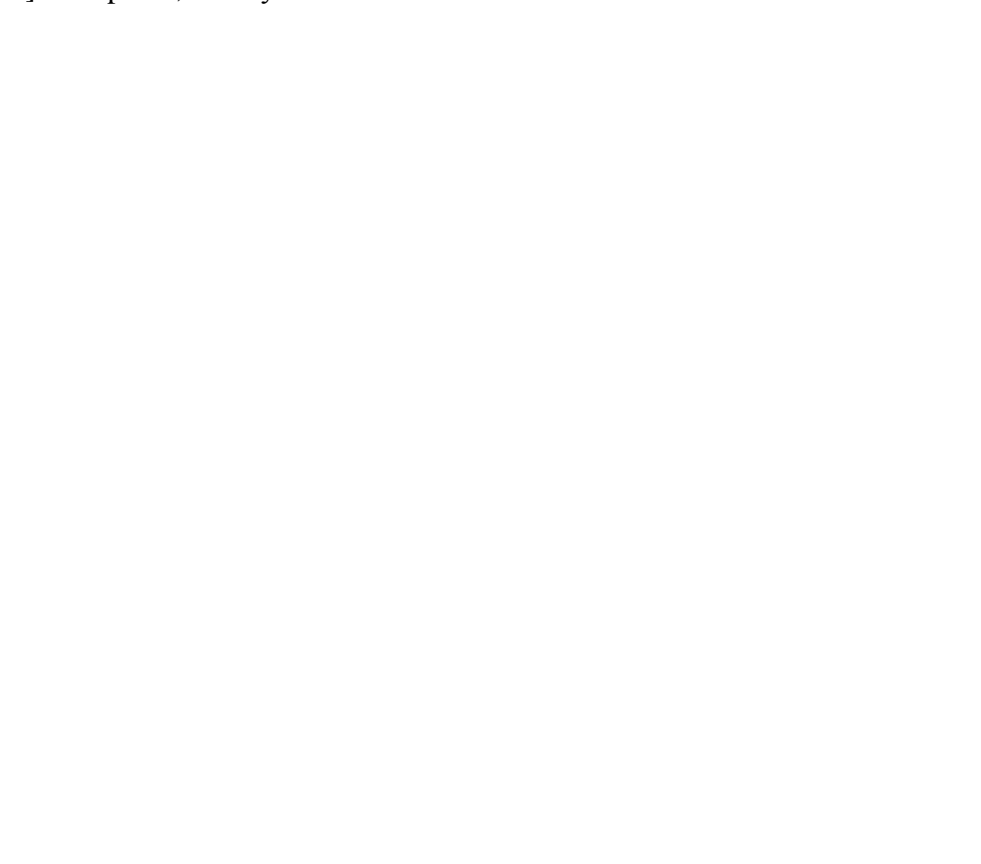

